\title{
A heterogeneous multi-criteria multi-expert decision-support system for scoring combinations of flood mitigation and recovery options
}

\author{
Fabio Zagonari ${ }^{\mathrm{a},}{ }^{\text {, }}$, Claudio Rossi ${ }^{\mathrm{b}}$ \\ a Dipartimento di Scienze Economiche, Università di Bologna, via Angherà 22, 47900 Rimini, Italy \\ ${ }^{\mathrm{b}}$ Università di Bologna, Italy
}

\section{A R T I C L E I N F O}

\section{Article history:}

Received 2 October 2012

Received in revised form

17 August 2013

Accepted 19 August 2013

Available online 19 September 2013

\section{Keywords:}

Multi-criteria multi-expert

Decision-support system

Flood mitigation

Flood recovery

.NET framework

\begin{abstract}
A B S T R A C T
In this study, we developed an innovative operational decision-support system (DSS) based on flood data and mitigation or recovery options, that can be used by both naïve and expert users to score portfolios of flood mitigation or recovery measures. The DSS combines exposure (i.e., economic, social, or environmental values at risk) and resilience (i.e., protection of the main equilibrium functions of human and physical systems). Experts from different fields define indices and functions, stakeholders express their attitudes towards risk, relative weights, and risk perceptions, and both groups use a shared learning process for risk assessment. The DSS algorithms include the "technique for order performance by similarity to ideal solution" (TOPSIS) and the "basic linguistic term set" (BLTS) methods for heterogeneous multi-criteria multi-expert decision-making. Decisions are illustrated using fixed or bounded values of flood depth, duration, and frequency, with plausible parameter values, for a case study of Cesenatico. The best mitigation option was construction of sand dunes and development of evacuation plans, which achieved $32 \%$ of the potential net benefit. The best recovery option was construction of sand dunes and development of evacuation plans and insurance schemes, which achieved $42 \%$ of the potential net benefit. Mitigation options outperformed recovery options whenever the relative importance of exposure with respect to resilience was greater than $95 \%$. Sensitivity analysis revealed that the best mitigation option was most robust with respect to flood duration and depth; the best recovery option was most robust with respect to the relative weights attached to economic, social, and environmental factors. Both options were similarly robust with respect to interdependencies between the options.
\end{abstract}

(c) 2013 Elsevier Ltd. All rights reserved.

\section{Introduction}

Due to the ever-increasing complexity of human society, people often need to consider multiple criteria (attributes, factors, objectives) to make decisions (Chen et al., 2011a). Multi-criteria decisionmaking (MCDM) is a response to the human inability to analyse multiple streams of heterogeneous information in a structured way: preferential information is modelled by weighting factors (i.e., inter-criteria comparisons) and value functions (i.e., intra-criteria preferences).

However, multi-disciplinary approaches are often needed, which require the involvement of experts from different areas, each with distinct knowledge and experience, and require different judgement and evaluation methods (e.g., qualitative and quantitative forms; certain and uncertain assessments). Because of the multiple disciplines, it is necessary to consider a range of at least partially conflicting objectives, such as economic vs. environmental

\footnotetext{
* Corresponding author. Tel.: +39 0541 434135; fax: +39 0541434120.

E-mail address: fabio.zagonari@unibo.it (F. Zagonari).
}

impacts or social vs. health aspects (Li et al., 2010). Multi-criteria multi-expert decision-making (MCMEDM) is a methodology to deal with the inherent complexity and uncertainty of such problems as well as the vague knowledge arising from the participation of many experts in the decision-making process (Yan et al., 2011). The main examples of MCMEDM models are crisp or fuzzy "techniques for order performance by similarity to ideal solutions" (TOPSIS; e.g., Chen et al., 2011b), the "basic linguistic term set" (BLTS) method (Herrera et al., 2005), the defuzzification centroid method (e.g., Vahdani et al., 2011), crisp or fuzzy linear programming techniques (e.g., Bereketli et al., 2011), the cross-entropy approach (e.g., Ye, 2011), possibility or probability approaches (e.g., Yuen and Lau, 2009), and geometric (e.g., Tan, 2011) or recursive (e.g., Tsiporkova and Boeva, 2006) or stochastic (e.g., Hahn and Knott, 2008) judgement aggregation models.

In addition, decision situations often involve stakeholders and expert groups with diverse background knowledge and different views, responsibilities, and interests, and in these situations, formally accredited experts are not necessarily the decision-makers (Yan et al., 2011). The rising demand for information by the mass 
media and the public often requires transparency and traceability of decisions, and must account for political and socio-psychological aspects (Roghanian et al., 2010). A multi-criteria multi-expert decision-support system (MCMEDSS) is a response to the need to improve communication and justify decision-making processes. This approach helps to form an audit trial that will enhance public confidence and understanding during and after the corresponding decision processes.

However, systematic non-linear multi-dimensional sensitivity analysis is not well supported by participation facilities (e.g., Bertsch et al., 2007), and software support has not yet been based on recent methodologies discussed in the literature on heterogeneous MCMEDM (e.g., Geldermann et al., 2009).

The purpose of the present study was to provide an innovative operational decision-support system (DSS) for both naïve users (with naïve defined based on their knowledge of the underlying methodology and their familiarity with the supporting software) and expert users (defined in terms of their knowledge and experience with the method and the supporting software). The DSS will be based on established procedures within the MCMEDM literature and used to achieve a score for each portfolio of flood mitigation options (MOs) and recovery options (ROs), with respect to both exposure (i.e., values at risk) and resilience (i.e., damage that will not alter the main equilibrium functions of the human and physical systems). In determining these scores, each group of experts specifies indices and functions, stakeholders express their attitudes towards risk, provide relative weights attached to economic, social and environmental issues, and describe their perceptions of risk, and the experts and stakeholders jointly participate in a shared learning process in which the risk assessment is based on the attitudes to and perceptions of risk.

Note that the DSS described in this paper is operational because it runs using real data from flood MOs and ROs in Cesenatico, Italy, a well-known tourist resort on the coast of the Northern Adriatic Sea. In particular, as an example of a DSS, it implements all "best practices" recommendations related to designing for ease of use and usefulness, establishing trust and credibility, and promoting acceptance of the DSS (McIntosh et al., 2011), as well as it performs all actions required to provide "good-quality" results (Van Delden et al., 2011). It also has the following recommended characteristics (Vahdani et al., 2011): it can be easily implemented using free software; it applies a simple and straightforward computation process; it is a vehicle for learning; it considers both the best and the worst alternatives concurrently; it performs an extensive visual and non-linear sensitivity and robustness analysis; and it depicts the best performance of the available alternatives in terms of each assessment criterion and in terms of the relative importance of objective and subjective information.

In terms of its involvement of end-users, it shares the available information between users and researchers; matches the perceptions, experiences, and operational procedures of the policymakers; and enhances the current policy practices by involving decision-makers at the municipality level as end-users during the development process in terms of identifying the long-term strategic issues to be focused on, the model assumptions, and data availability. Although it is informed by all relevant interests and stakeholders, it does not adopt a participatory modelling approach, which would aim at achieving "wider social change through the democratisation of decision-making" (Van Delden et al., 2011). However, the speed is sufficiently fast that the software is appropriate for use in interactive workshop sessions, together with individual decision simulations.

In terms of the success of its adoption, it accomplishes a task that end-users are already required to do by providing new, reliable, relevant, complete, and reliable information to improve the effectiveness and efficiency of actions, while allowing users and stakeholders to reproduce the decision procedure, play with the weights and parameters, and perform a sensitivity analysis to assess the strength and robustness of decisions. However, it is not built on psychological and organisational theory, and it is difficult to evaluate its impacts on attitudes, behaviours, and learning "to build social capital between individuals" (McIntosh et al., 2011).

As an example of an environmental DSS, it represents the complex interactions that take place in the human-environment system (Van Delden et al., 2011) by informing environmental risk management (McIntosh et al., 2011).

The DSS we will describe is innovative because it incorporates (within its algorithms) the TOPSIS and BLTS methods from the literature on heterogeneous MCMEDM while measuring (in its results) the concepts of exposure, vulnerability, sensitivity, resilience, and susceptibility from the flood risk literature. In particular, as an example of a DSS, it integrates models, incorporates data, and processes knowledge from different disciplines (Van Delden et al., 2011). As a result, it improves the consistency and the quality of decisions (McIntosh et al., 2011) in a complex and ill-structured domain that is characterised by a large of number of actors, a high level of uncertainty, and a large number of conflicting interests. As an example of an environmental DSS, it bridges the gap that is inherent in ecological, social, and economic models and that arises from different languages, scales, and paradigms (Van Delden et al., 2011). It does so by bringing together multiple stakeholders to learn from each other and jointly assess the impacts of management options (McIntosh et al., 2011), with strong concern for the transparency of the underlying assumptions.

However, as an example of a semi-structured DSS, which aims at identifying the need for change (i.e., the intelligence-gathering phase), evaluating alternatives, and choosing (i.e., the choice phase) but not designing alternative options, it cannot be extended by adding modules (McIntosh et al., 2011). As an example of a strategic-purposed environmental DSS, which combines database and modelling perspectives from engineering, economics, sociology, and ecology to facilitate decision-making, it calculates endconditions rather than simulating temporal dynamics (Van Delden et al., 2011).

\section{The decision-support system}

The purpose of this section is twofold. First, we describe the situation for Cesenatico, Italy, which will be used as a case study to demonstrate our approach, together with some assumptions related to that context. Second, we combine some of the consistent procedures presented in Supplementary material I and II with some simplifying assumptions.

\subsection{The case study}

Four main sources of flooding have been identified for Cesenatico: waves, storms and tides; run-off and river discharge; subsidence due to water and gas extraction; and sea level rise due to climate change. Erosion could also have been discussed in another context, but it is not highly relevant for Cesenatico. Our analysis considers three flood characteristics: depth, duration, and frequency. Velocity and whether the flooding is permanent could also have been discussed, but these factors were not relevant for Cesenatico.

In our analysis, we consider three flood mitigation and recovery options: constructing sand dunes, developing evacuation plans, and designing insurance schemes. From an economics perspective, an insurance scheme will be shown to be equivalent to a subsidised loan. Spatial planning, the installation of sea walls, beach 
nourishment, managed realignment of the shoreline and upgrading existing defences could also have been discussed, but they are less relevant for Cesenatico.

Note that in our model, insurance schemes (an example of a mitigation option) are assumed to be compulsory, without exemptions, and are depicted by a premium that represents a proportion of the average yearly exposure, whereas loan subsidies (an example of a recovery option) are depicted as interest rates calculated based on an expected yearly exposure and as a fixed repayment required to pay back the yearly exposure over a number of years that depends on the expected flood frequency. Loan subsidies satisfy the condition that interest plus annuities are less than $1 / 5$ of the average yearly income. Evacuation plans (an example of mitigation and recovery options) are assumed to alleviate psychological distress, which is qualitatively measured in terms of the effects on health and of social distress. The construction of sand dunes (an example of a mitigation option) is measured in terms of the expected reduction in flood depth and duration, and disregard climate change scenarios.

Land uses (ha) in Cesenatico (Beach 54.80, Residential home 48.51, Holiday home 26.25, Historical building 32.07, Hotel 52.49, Camping site 20.18, Tourism harbour or infrastructure 6.32, Fishing harbour or infrastructure 5.82, Private service 7.99, Sport or social infrastructure 18.81, Hospital 1.53, School or social service 7.42, Public infrastructure 116.49, Public or private environmental resource 69.09) suggest that several economic, social, and environmental features are relevant in our analysis. Economic damage to property, employment reduction, and landscape degradation will be considered to represent potential economic exposure. Species (i.e., here key species, not a proxy for habitat, since they are not habitat formers) and habitat degradation will be considered to represent potential ecological exposure. Health and social distress will be considered to represent potential social exposure.

\subsection{Contextual assumptions}

Based on our knowledge of the study area and our description in Section 2.1, we proposed the following six contextual assumptions:

1. Four groups of experts $(j)$ are relevant: engineers, economists, ecologists, and sociologists. The engineers provide information to the other experts to support their assessment of impacts (e.g., construction costs for economists, environmental impacts for ecologists). This approach avoids double-counting. In our case study, economists were assumed to apply real numbers to economic damages and costs, and linguistic labels to impacts on employment and landscape. In contrast, ecologists would use interval numbers for consequences on species and habitats, and sociologists would apply linguistic labels for types of social and health distress.

2. Experts within a given group are homogeneous with respect to their criteria, attributes, weights, granularity of linguistic labels, and so on. As a result, each group of experts $(j)$ expresses a single opinion on each criterion $(i)$ and attributes the same weight to that criterion $\left(E_{i}\right)$.

3. Each group of experts $(j)$ combines crisp preferences or indices for $X_{i j}$ using one or more alternative forms (e.g., [0,1] for real numbers, $(-\infty,+\infty)$ for real numbers, interval numbers, or linguistic labels) for each criterion or attribute $(i)$ and weights attached to that criterion $\left(E_{i}\right)$ in order to achieve a score for each alternative or portfolio of mitigation and recovery options $(k)$, where $X_{i j}$ depends on the flood depth, duration, and frequency.

4. A hierarchical decision-making structure is adopted so that each set of criteria or attributes $(i)$ is peculiar to group of experts $j$ : in other words, an economic criterion (e.g., employment) cannot be found in social and ecological features, and similarly for other criteria. Weights attached to feature $S_{j}$ (economic, social, or environmental) are only specified by stakeholders.

5. DSS users can select a combination of mitigation and recovery options: this implies that even though the criteria $(i)$ are identified by each group of experts $(j)$ independently from other groups of experts, preferences or indices for several criteria for each alternative must account for potential interactions or interdependencies between the impacts of all possible combinations of alternatives (e.g., an evacuation plan could be less effective if coupled with sand dune creation, because people could become less involved in evacuation simulations). We will apply an interaction and interdependence matrix to resolve this problem.

6. Scoring is the goal (i.e., score $a>$ score $b>$ score $c>$ score $d$ ) within a satisficing approach (i.e., "choose an alternative as the best if its performance is at least good as a specified target"). Alternative methods could be used, such as choosing, which could be depicted by $a>\{b, c, d\}$, sorting by saying that $\{a, b\}$ is preferred to $\{c, d\}$, and ranking by stating that $a$ is preferred to $b$ is preferred to $c$ is preferred to $d$.

Some observations are relevant here. First, the assumption of homogeneity of the experts within each group (assumption 2) avoids the need to use the fuzzy analytical hierarchy process (e.g., Mikhailov and Tsvetinov, 2004), a fuzzy genetic algorithm (e.g., Su and Wang, 2012), or a Pareto set (e.g., Dowhan et al., 2009) to combine different criteria, attributes, or weights. This makes the analysis of different granularities of linguistic labels irrelevant (e.g., Li et al., 2010), and suggests that the use of a last-aggregation approach would be more precise, since there is no variation among experts within a group (e.g., Roghanian et al., 2010). This approach favours a focus on aggregation by disregarding the identification of a consensus process to resolve conflicts between experts (e.g., Guha and Chakraborty, 2011; Parreiras et al., 2010; Ekel et al., 2009).

Second, the absence of vagueness in the preferences expressed by experts within each group (assumption 3 ) suggests the application of fuzzy sets only to combine preferences or indices in alternative forms, such as the BLTS method suggested by Herrera et al. (2005) and applied by Halouani et al. (2009), rather than using fuzzy sets to depict uncertainty, by avoiding the need to use the defuzzification centroid method (e.g., Vahdani et al., 2011). It would also be possible to apply possibility or probability approaches (e.g., Yuen and Lau, 2009), or to use geometric (e.g., Tan, 2011), recursive (e.g., Tsiporkova and Boeva, 2006), or stochastic judgment (e.g., Hahn and Knott, 2008) aggregation models.

Third, the hierarchical decision-making structure we adopted (assumption 4) implies that the BLTS method is applied to combine different opinion forms within each group of experts (e.g., Halouani et al., 2009) rather than using different opinions by different alternative experts (e.g., Chu et al., 2007).

Fourth, the use of a potential interaction and interdependence matrix between preferences or indices (assumption 5) rather than between criteria (e.g., Dalalah et al., 2011) implies the use of arithmetic aggregation by disregarding geometric aggregation (e.g., Tan, 2011) or conflicting criteria (e.g., Wu, 2009).

Note that DSS users (either stakeholders or single groups of experts) are expected to specify the flood depth, duration, and frequency, which are the three main items used to depict risk perception. Unlike existing multi-criteria multi-expert DSS (e.g., Geldermann et al., 2009), which were based on multi-attribute value theory, heterogeneity of information is taken into account. 
Finally, experts are expected to specify impact functions rather than to carry out pair-wise comparisons or specify a decision information matrix (e.g., Anisseh et al., 2012). To improve transparency (e.g., Kain and Soderberg, 2008), DSS users can specify new parameters or weights or accept the previously recorded parameters or weights, thereby letting stakeholders and single groups of experts independently perform numerical simulations.

In other words, we have applied a multi-criteria decision analysis approach in which relative weights are specified by the stakeholders, the TOPSIS method is used to normalise group values, and crisp interval values or linguistic labels are made uniform by applying the BLTS method (see Supplementary material II). In this way, we develop a heterogeneous multi-expert multi-criteria DSS by disregarding fuzzy and group decision-making. Since choosing an urban area for our case study means that the probability of flooding (i.e., a hazard) amounts to the probability of harm (i.e., a susceptibility), we have defined exposures as the potential damage and resilience as the magnitude of damage that will not alter the main functions under equilibrium conditions (static version) of human and physical systems (see Supplementary material I). We have performed this definition for discrete times and at a very local scale, and have deduced vulnerability from exposure and resilience (i.e., vulnerability $=$ exposure - resilience), and have obtained sensitivity from susceptibility and vulnerability (i.e., sensitivity = susceptibility $\times$ vulnerability).

\subsection{Simplifying assumptions}

\section{We made four simplifying assumptions:}

1. We used the Euclidian distance, since experts are assumed to have clear ideas about distances as well as about the positions of the best and worst points. An alternative would be to use membership functions for measuring distances (e.g., Anisseh et al., 2012) or non-linear programming to identify the best and worst points (e.g., Li et al., 2010).

2. We applied absolute distances within the TOPSIS method because this approach is readily comprehensible for users who express a degree of risk aversion. Alternatively, relative distances (e.g., Vahdani et al., 2011), constant elasticity of substitution aggregations (e.g., Chamodrakas and Martakos, 2011), or similarity to the extreme solutions (e.g., Dheena and Mohanraj, 2011) could be applied.

3. We used arithmetic averages to emphasise the multidisciplinarity of the approach. An alternative would be geometric averages that stress each individual feature (e.g., Ye, 2011).

4. We used triangular fuzzy numbers to aggregate heterogeneous preferences, since experts are assumed to have clear ideas about values. Alternatively, trapezoidal fuzzy numbers (e.g., Liu, 2011) could be applied.

In other words, for each option/alternative $k, \sum_{j} S_{j}[Y$ $\left.D_{\mathrm{B}, j k}+(1-Y) D_{\mathrm{W}, j k}\right]=X_{k}$ within $[0,1]$ is calculated, with

$$
\begin{aligned}
& D_{\mathrm{B}, j k}=\sqrt{ } \sum_{i} E_{i j}\left(X_{i j k}-B_{i j}\right)^{2} \\
& D_{\mathrm{W}, j k}=\sqrt{ } \sum_{i} E_{i j}\left(X_{i j k}-W_{i j}\right)^{2}
\end{aligned}
$$

In these equations, $X_{i j k}$ (a function of flood depth, duration, and frequency specified by stakeholders or experts) are preferences or indices in crisp real numbers or interval numbers within $[0,1]$ or linguistic labels in $\{\mathrm{N}, \mathrm{L}, \mathrm{M}, \mathrm{H}, \mathrm{P}\}$, where $\mathrm{N}$ represents Null, $\mathrm{L}$ represents Low, $\mathrm{M}$ represents Medium, $\mathrm{H}$ represents High, and $\mathrm{P}$ represents Perfect. $S_{j}$ is within $[0,1]$, where $\sum_{j} S_{j}=1$ and represents the sum of the relative weights attached to features by stakeholders. $E_{i j}$ is within [0,1], where $\sum_{i} E_{i j}=1$ and represents the sum of the relative weights attached to the criteria by experts $(j)$ or stakeholders. $D_{\mathrm{B}, j k}$ and $D_{\mathrm{W}, j k}$ are the Euclidian distances from the best $\left(B_{i j}\right)$ and the worst $\left(W_{i j}\right)$ cases, respectively. $Y$ is the relative weight attached to the distances from the best cases. Similar reasoning applies to the resilience $R_{i j k}$, where the best and worst cases are set at 1 and 0 , respectively.

Note that information in different forms $\left(X_{i j k}\right)$ is transformed into fuzzy sets $\gamma_{h i j k}$ for reference index $h$, using the BLTS method suggested by Herrera et al. (2005). In particular, if $h=1,2,3,4$, and 5 are the reference indices, subscript $k$ is disregarded, and $\theta$ is the value specified by experts, then in the case of real numbers within $[0,1]$ :

$$
\begin{aligned}
& \gamma_{1 i j}=1, \gamma_{2 i j}=0, \gamma_{3 i j}=0, \gamma_{4 i j}=0, \text { and } \gamma_{5 i j}=0 \text { if } \theta=0 \\
& \gamma_{1 i j}=(\theta-0) /(0.25-0), \gamma_{2 i j}=(\theta-0) /(0.5-0), \gamma_{3 i j}=0, \\
& \gamma_{4 i j}=0, \text { and } \gamma_{5 i j}=0 \text { if } \theta \in(0,0.25] \\
& \gamma_{1 i j}=0, \gamma_{2 i j}=(\theta-0) /(0.5-0), \gamma_{3 i j}=(\theta-0.25) /(0.75-0.25), \\
& \gamma_{4 i j}=0, \text { and } \gamma_{5 i j}=0 \text { if } \theta \in(0.25,0.5] \\
& \gamma_{1 i j}=0, \gamma_{2 i j}=0, \gamma_{3 i j}=(\theta-0.25) /(0.75-0.25), \\
& \gamma_{4 i j}=(\theta-0.5) /(1-0.5), \text { and } \gamma_{5 i j}=0 \text { if } \theta \in(0.5,0.75] \\
& \gamma_{1 i j}=0, \gamma_{2 i j}=0, \gamma_{3 i j}=0, \gamma_{4 i j}=(\theta-0.5) /(1-0.5), \text { and } \\
& \gamma_{5 i j}=(1-\theta) /(1-0.75) \text { if } \theta \in(0.75,1) \\
& \gamma_{1 i j}=0, \gamma_{2 i j}=0, \gamma_{3 i j}=0, \gamma_{4 i j}=0, \text { and } \gamma_{5 i j}=1 \text { if } \theta=1
\end{aligned}
$$

If $h=1,2,3,4$, and 5 are the reference indices, subscript $k$ is disregarded, and $\left[\theta_{L}, \theta_{U}\right]$ are the lower and upper bounds of the intervals specified by experts, respectively, then in the case of interval numbers within $[0,1]$ :

$\gamma_{1 i j}=0.5, \gamma_{2 i j}=0.5, \gamma_{3 i j}=0, \gamma_{4 i j}=0$, and $\gamma_{5 i j}=0$ if $\left[\theta_{L}, \theta_{U}\right]=[0,0.25]$

$\gamma_{1 i j}=0, \gamma_{2 i j}=0.5, \gamma_{3 i j}=0.5, \gamma_{4 i j}=0$, and $\gamma_{5 i j}=0$ if $\left[\theta_{L}, \theta_{U}\right]=[0.25,0.5]$

$\gamma_{1 i j}=0, \gamma_{2 i j}=0, \gamma_{3 i j}=0.5, \gamma_{4 i j}=0.5$, and $\gamma_{5 i j}=0$ if $\left[\theta_{L}, \theta_{U}\right]=[0.5,0.75]$

$\gamma_{1 i j}=0, \gamma_{2 i j}=0, \gamma_{3 i j}=0, \gamma_{4 i j}=0.5$, and $\gamma_{5 i j}=0.5 \operatorname{if}\left[\theta_{L}, \theta_{U}\right]=[0.5,1]$

If $h=\mathrm{N}, \mathrm{L}, \mathrm{M}, \mathrm{H}$, and $\mathrm{P}$ are the reference indices, subscript $k$ is disregarded, and $\theta$ is the label specified by the experts, then in the case of linguistic labels:

$\gamma_{1 i j}=1, \gamma_{2 i j}=0, \gamma_{3 i j}=0, \gamma_{4 i j}=0$, and $\gamma_{5 i j}=0$ if $\theta=\mathrm{N}$
$\gamma_{1 i j}=0, \gamma_{2 i j}=1, \gamma_{3 i j}=0, \gamma_{4 i j}=0$, and $\gamma_{5 i j}=0$ if $\theta=\mathrm{L}$
$\gamma_{1 i j}=0, \gamma_{2 i j}=0, \gamma_{3 i j}=1, \gamma_{4 i j}=0$, and $\gamma_{5 i j}=0$ if $\theta=\mathrm{M}$
$\gamma_{1 i j}=0, \gamma_{2 i j}=0, \gamma_{3 i j}=0, \gamma_{4 i j}=1$, and $\gamma_{5 i j}=0$ if $\theta=\mathrm{H}$
$\gamma_{1 i j}=0, \gamma_{2 i j}=0, \gamma_{3 i j}=0, \gamma_{4 i j}=0$, and $\gamma_{5 i j}=1$ if $\theta=\mathrm{P}$

Moreover, these data are transformed into values within $[0,1]$ by applying $X_{i j k}=\sum_{j=0}^{\mathrm{H}} h \gamma_{h i j k} / \sum_{j=0}^{\mathrm{H}} \gamma_{h i j k}$ (see Herrera et al., 2005, for general formulations). Here, no semantic overlapping is considered. Finally, the interval numbers expressed by the experts are assumed to coincide with the specified intervals $\left[\theta^{L}, \theta^{U}\right]$ (see Herrera et al., 2005, for general cases).

\section{The software}

The purpose of this section is twofold. First, we illustrate the software architecture, by highlighting its potentials: detailed installation procedures are presented in Supplementary material III. Second, we describe the software interface, by stressing the main advantages with respect to existing software: additional details about buttons and functions are provided in Supplementary material IV. 


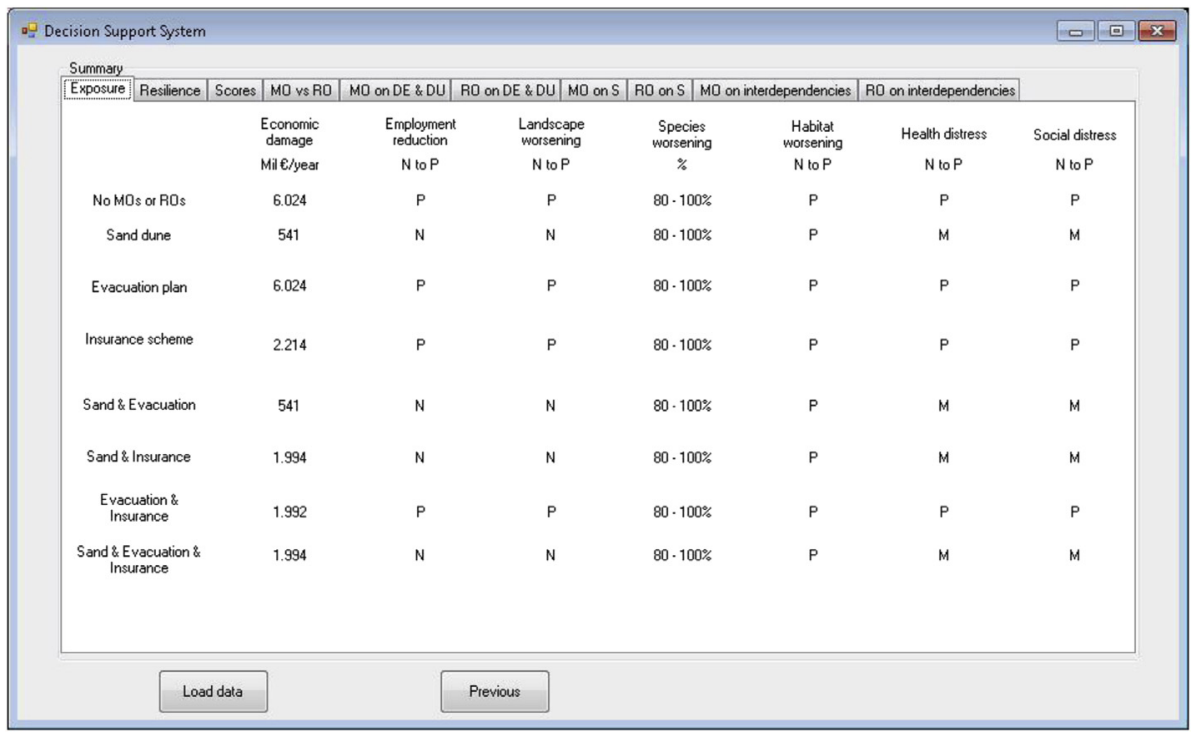

Fig. 1. Summary of exposure in unalike forms as a function of flood depth, duration, and frequency.

\subsection{The software architecture}

The equations discussed in the previous sections were implemented as an original program that we named "MC-ME-DSS". The MC-ME-DSS architecture consists of three internal elements: a graphical user interface (GUI); a wrapper for calculations; and a database input output system (DBIOS). From an external perspective, the GUI of the DSS allows the user to insert input data (e.g., weights, values) and to obtain output results (e.g., scores, graphics). Numerical values are accepted as inputs within a specified range, and they are redisplayed as they are stored: the data format and type coincide. From an internal perspective, the GUI of the DSS is independent of the wrapper and the DBIOS (i.e., it is not integrated with either of those two programs). Specifically, it loads input data from the DBIOS and it stores output data in the DBIOS by recording the date, hour, and minute of the generation of output data. It sends calculation requests in Wolfram's Mathematica language to the wrapper, although it could use other languages (e.g., Mathworks' MatLab), and it receives the calculation results from the wrapper.

Note that the user only has access to the GUI. Moreover, all elements in the DSS (i.e., the GUI, wrapper, and DBIOS) are coded using the .NET framework. In general, this framework allows the development of platform-independent applications (i.e., programs that will run under any operating system that supports the .NET framework). Thus, it's not necessary to develop alternative versions of the DSS for different platforms. In other words, the highest degree of platform compatibility is achieved. Finally, the wrapper and the DBIOS are insulated from each other so that they can be updated independently.

The MC-ME-DSS architecture is supported by three external elements: an operating system (e.g., Windows versions from XP to 8 , Linux); Microsoft SQL Server; and Wolfram's Mathematica kernel. The DBIOS sends output results to Microsoft SQL Server and it loads scenarios (i.e., input and output datasets) from Microsoft SQL Server. Any ODBC-compliant software (e.g., Microsoft Excel, OpenOffice (alc) can be used to read and process the output results (e.g., to perform statistical analysis) as well as to insert and process the input data (e.g., to carry out simulations within the DSS using a previously defined set of scenarios). In other words, the maximum degree of exportability and importability of data and results is obtained.
The wrapper maps the calculation requests received from the GUI to the functions provided by the Mathematica kernel and it receives numbers, graphics, and error messages as outputs. Many calculation programs can be used, since the wrapper uses abstract forms compatible with the language and logic of the Mathematica kernel. In other words, the highest degree of software applicability is achieved.

Both Microsoft SQL Server and the Wolfram Mathematica kernel are coded in their native language: the wrapper will only need to be updated if the Mathematica kernel changes or if alternative software is used for the calculations. Similarly, the use of an alternative database management system would only require updating of the DBIOS.

The following components are required for installation of MCME-DSS: the Wolfram Mathematica kernel or the Wolfram CDF Player, Microsoft SQL Server, and .NET Framework 2.0. Mathematica can be bought from Wolfram (www.wolfram.com/mathematica), but the Wolfram CDF Player is a free download (www.wolfram. com/cdf-player). Microsoft SQL Server can be bought from Microsoft, but a free version is also available (SQL Server Express; www. microsoft.com/en-us/sqlserver/editions/2012-editions/express. aspx). The .NET framework for Windows can be freely downloaded (msdn.microsoft.com/en-us/vstudio/aa496123.aspx). Version 2.0 of the .NET framework is already included in Windows Vista and subsequent versions. Thus, a high degree of software availability is obtained.

Supplementary material III describes how to install MC-ME-DSS. The DSS is an executable file. However, since it is supported by three external elements, it was not possible for us to implement a complete installer program; although installing the framework is not a problem, Microsoft SQL Server and Wolfram's Mathematica kernel both require the implementation of specific procedures that depend on the computer where the DSS package will be installed. However, the installation procedure is not difficult, so a high degree of software applicability is achieved.

\subsection{The software interface}

In the software, interface 1 is expected to be used for inputs by both stakeholders and experts. Interfaces 2 to 5 will be used for inputs by economists, engineers, ecologists, and sociologists, 


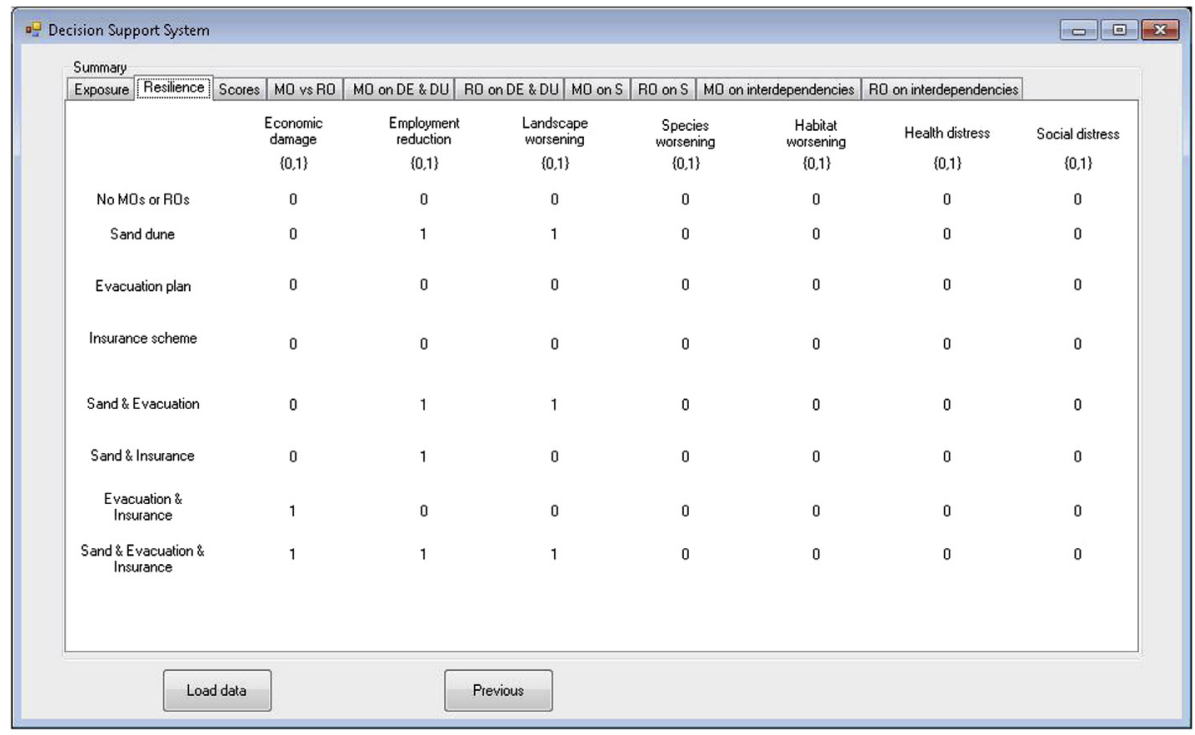

Fig. 2. Summary of resilience in unalike forms as a function of flood depth, duration, and frequency.

respectively. Interface 6 will be used for inputs by experts to define the costs per year (including construction, operating, and maintenance costs) and relevant interdependencies (see Supplementary material IV). Interfaces 7-10 (Figs. 1-4) will be used to present numerical and graphical results, while interfaces 11-16 (Figs. 5-10) will be used to present sensitivity analyses.

Relative weights by stakeholders $\left(S_{j}\right)$ and by experts $\left(E_{i}\right)$ are introduced as parameters, but they could instead be estimated as linguistic labels or as real numbers, as suggested by Kodikara et al. (2010). In this particular example, the average values of $S_{1}$ (i.e., the relative weight attached to economic issues), $S_{2}$ (i.e., the relative weight attached to ecological features), and $S_{3}$ (i.e., the relative weight attached to social issues) are $0.23,0.58$, and 0.19 , respectively. Flood frequency $(F R)$ is measured as the expected number of years between floods. It measures the perceived probability of a damaging flood and depicts the risk perception after subtracting preparedness. We applied the absolute distances from the best cases $(Y)$ and the worst cases $(1-Y)$, although alternative approaches could have been used (see Vahdani et al., 2011; Chamodrakas and Martakos, 2011; Dheena and Mohanraj, 2011).

The following functions are applied for exposure $X$, where the second subscript $(j)$ refers to the expert groups (i.e., 1 for economists, 2 for ecologists, and 3 for sociologists), while the first subscript $(i)$ refers to relevant categories for each expert group (i.e., 3 categories for economists: economic damages, employment and landscape; 2 categories for ecologists: species and habitats; and 2 categories for sociologists: social and health distress)

- Economists:

Real numbers: $X_{11}=\sum_{u} v_{u} b_{u}(1-S U) D U a_{u} \sqrt{ }[(1-\mathrm{SE})$ $D E]+C O$ with $B_{11}=0$ and $W_{11}=\sum_{u} v_{u} b_{u} 30 a_{u} \sqrt{ } 1.5$, where $v, b$ and $a$ represent the economic values $(€ /$ ha), the damage (\%) at the maximum flood depth, the damage (\%) at the maximum flood duration, respectively, $\mathrm{CO}$ depicts the cost of mitigation options, and subscript $u$ stands for (land) use

$O$ Linguistic labels: $X_{21}=\mathrm{N}$ if $(1-S E) D E<T_{21,1}, X_{21}=\mathrm{L}$ if $(1-S E) D E$ is within $\left[T_{21,1}, T_{21,2}\right], \ldots, X_{21}=\mathrm{P}$ if $(1-S E)$ $D E>T_{21,4}$, where $T$ stands for Threshold

$O$ Linguistic labels: $X_{31}=\mathrm{N}$ if $(1-S E) D E<T_{31,1}, X_{31}=\mathrm{L}$ if $(1-S E) D E$ is within $\left[T_{31,1}, T_{31,2}\right], \ldots, X_{31}=\mathrm{P}$ if $(1-S E)$ $D E>T_{31,4}$
- Ecologists:

Interval numbers: $X_{12}=0-20 \%$ if $D E<T_{12,1}, 20-40 \%$ if $D E$ is within $\left[T_{12,1}, T_{12,2}\right], \ldots X_{12}=80-100 \%$ if $D E>T_{12,4}$, with $B_{12}=0 \%$ and $W_{12}=100 \%$

$\bigcirc$ Linguistic labels: $X_{22}=\mathrm{N}$ if $D U<T_{22,1}, X_{22}=\mathrm{L}$ if $D U$ is within $\left[T_{22,1}, T_{22,2}\right], \ldots, X_{22}=\mathrm{P}$ if $D U>T_{22,4}$

- Sociologists:

Linguistic labels: $X_{13}=\mathrm{N}$ if $(1-S U) D U<T_{13,1}, X_{13}=\mathrm{L}$ if $(1-S U) D U$ is within $\left[T_{13,1}, T_{13,2}\right], \ldots, X_{13}=\mathrm{P}$ if $(1-S U)$ $D U>T_{13,4}$

Linguistic labels: $X_{23}=\mathrm{N}$ if $(1-S U) D U<T_{23,1}, X_{23}=\mathrm{L}$ if $(1-S U) D U$ is within $\left[T_{23,1}, T_{23,2}\right], \ldots, X_{23}=\mathrm{P}$ if $(1-S U)$ $D U>T_{23,4}$

- Engineers specify percentage impacts on flood depth $(D E)$ and flood duration $(D U)$ of a sand dune (SE and $S U$, respectively)

The following functions are applied for resilience $(R)$ :

- For $i=1$ and $j=1, R_{11}=1$ if $X_{11} / F R<0.2 \sum_{u} v_{u}$, but $R_{11}=0$ otherwise

- For $i \neq 1$ or $j \neq 1, R_{i j}=1$ if $X_{i j}<R T_{i j} I M$ and $F R>R T_{i j} F R$, but $R_{i j}=0$ otherwise, where $R T_{i j} I M$ and $R T_{i j} F R$ are thresholds on impacts and frequencies, respectively.

Note that resilience is measured by specifying the maximum exposure the economic or social or ecological system can absorb without disrupting its static equilibrium, with its dependence on time being depicted as the maximum tolerable frequency of exposure. Here, economic resilience refers to a heterogeneous-agent (individual) approach, whereas social and ecological resilience refer to a representative-agent (population) approach. Moreover, resilience is assumed to be a qualitative $\{0,1\}$ variable, with no reference to recovery periods, recovery percentages or levels, or recovery probabilities. An ecological status different from the status quo could be depicted as the best state by properly choosing the best and worst cases $\left(B_{12}, W_{12}, B_{22}, W_{22}\right)$ and thresholds $\left(T_{12}, T_{22}\right)$ for species and habitat indices. Finally, both exposure and resilience refer to the same time horizon, whether in the near or distant futures, and they take into account both direct and indirect impacts.

The construction of sand dunes (which affect flood pathways) and the development of evacuation plans (which affect flood 


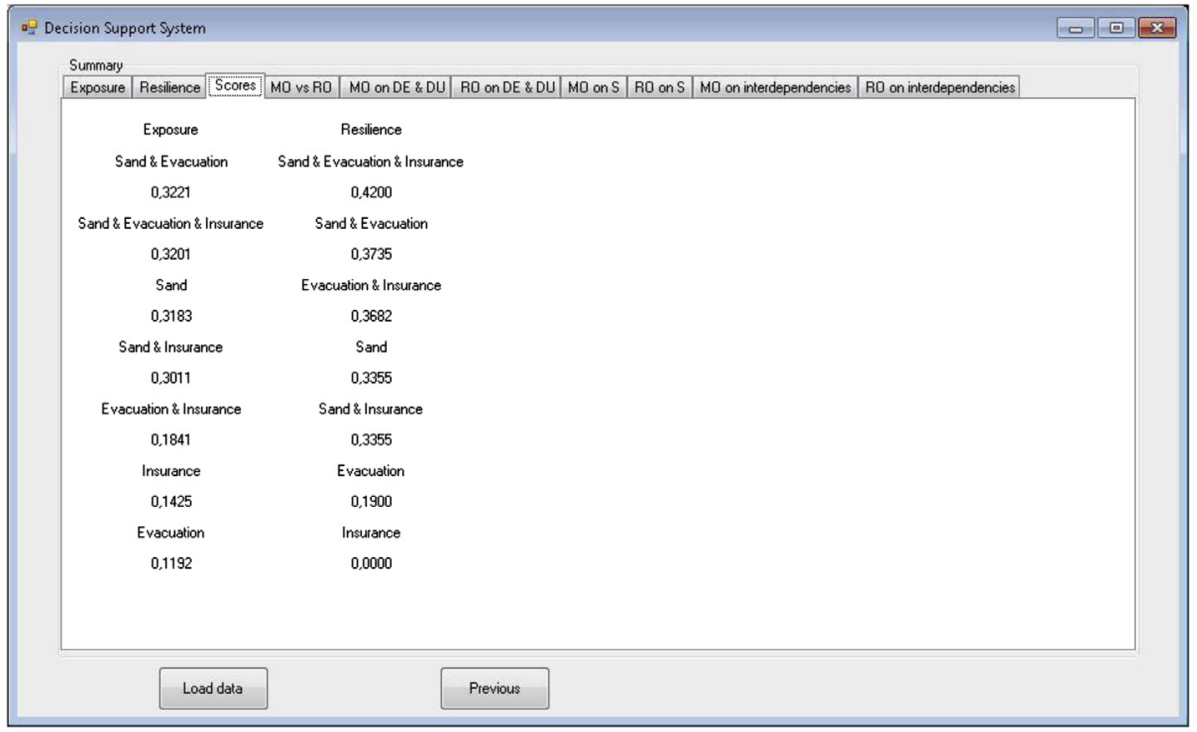

Fig. 3. Ordered scores within $[-1,1]$ for mitigation and recovery options from alike and combined information, in the short-run $X$ (exposure) and long-run $R$ (resilience).

receptors) are depicted as percentage reductions in exposure, whereas insurance schemes (which affect flood receptors) are represented as $I P \times X_{11} / F R$ and loan subsidies as $\left[L A \times X_{11} /\right.$ $F R]+\left[X_{11} \times I R\right]$, where IP and LA are the insurance premium and the loan annuity as percentages of the expected exposure, respectively. Insurance schemes and evacuation plans represent social innovation (i.e., new social processes adopted by society so that people can change their interactions), where evacuation plans affect resilience through increased awareness (a positive $e_{11} r$, where $e$ stands for evacuation plans, subscripts $i=1$ and $j=1$ identify the economic features, and $r$ stands for resilience) and insurance schemes affect exposure through increased preparedness (a positive $i_{11} x$, where $i$ stands for insurance schemes, subscripts $i=1$ and $j=1$ identify the economic features, and $x$ stands for exposure). The construction of sand dunes and design of loan subsidies represent technical innovation (i.e., new instruments for the municipality), where sand dunes affect exposure and loan subsidies affect resilience. Interdependencies between mitigation and recovery options are depicted by the reduced effectiveness of evacuation plans in terms of social and health distress if they are combined with the construction of sand dunes $\left(\mathrm{se}_{13} X\right.$ and $\mathrm{se}_{23} X$, where se stands for sand dunes combined with evacuation plans, subscripts $i=1$ with $j=3$ and $i=2$ with $j=3$ identify the social and health features, respectively, and $x$ stands for exposure).

Note that different mitigation and recovery options have different time scales: each option is assumed to refer to an average year for that option, with total costs being discounted but without considering cost distributions between affected groups, the municipality, the region, and the state. Moreover, mitigation and recovery options depend on flood histories and could evolve over time as these histories change. For simplicity, we assumed that they were fixed over time, with costs including operating and maintenance expenditures. Finally, different mitigation and recovery options have different spatial scales. For simplicity, we analysed a

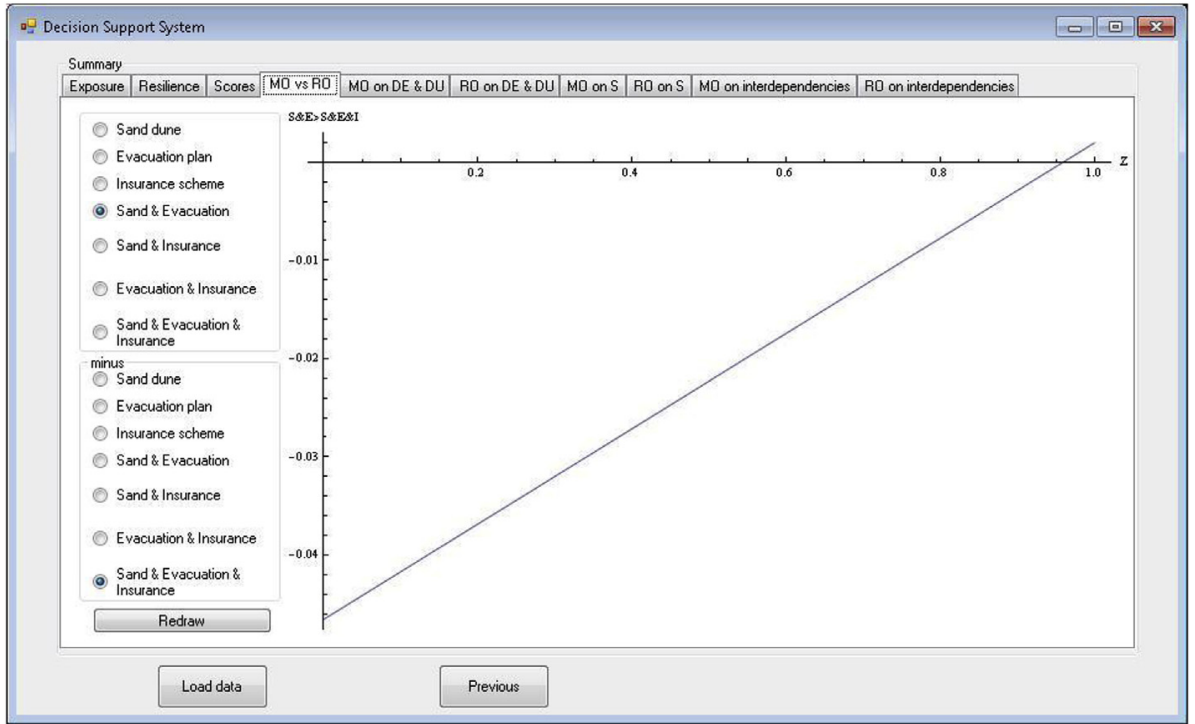

Fig. 4. Overall comparisons of the mitigation (Sand \& Evacuation) and recovery (Sand \& Evacuation \& Insurance) options with the best scores. 


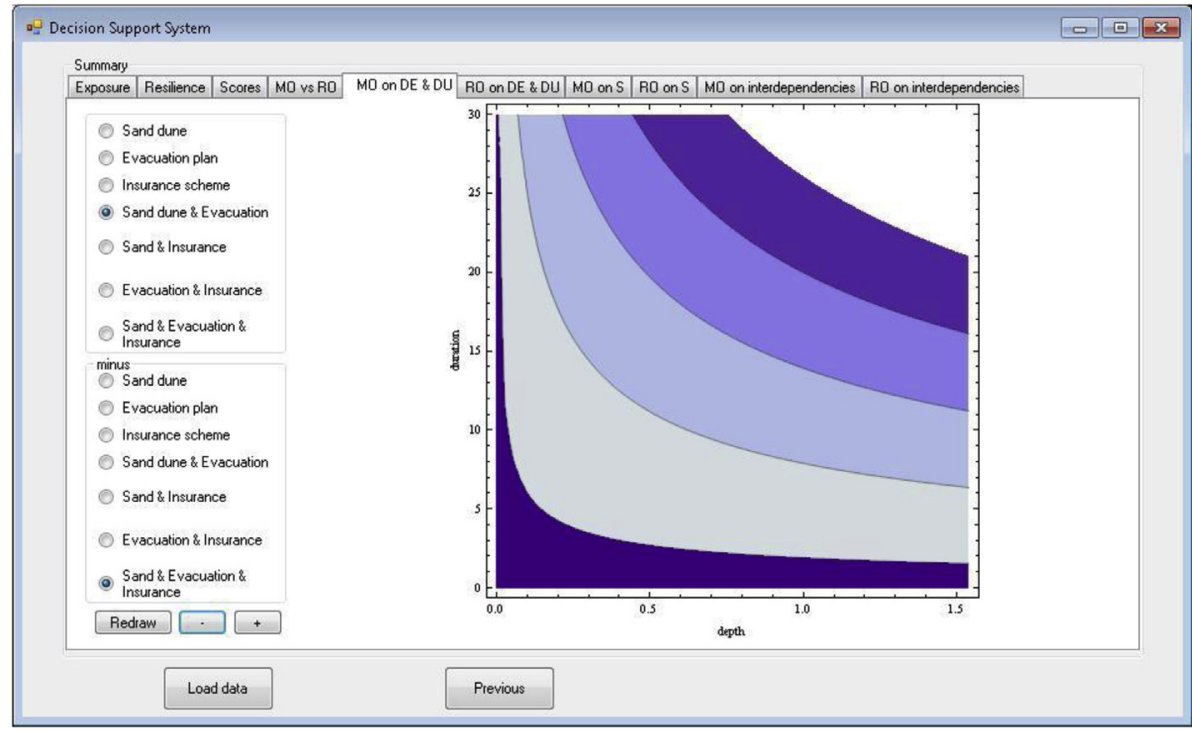

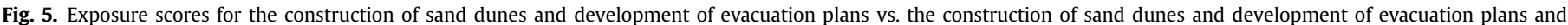
insurance schemes, both as a function of flood depth ( $\mathrm{m})$ and duration (days).

single area, since our case study focused on a single source (i.e., the sea) and a single receptor (i.e., the municipality), without applying a nested approach for different areas.

The results of the model's numerical simulations are saved in a dataset. This allows users of the software to perform static and dynamic analyses of the decisions taken and of the values expressed by stakeholders and experts.

Note that we assumed the numerical simulations would be jointly performed by experts and stakeholders, within a participatory planning approach. This assumption has three main implications. First, there are no pre-cooked scenarios: the most relevant changes can be directly depicted rather than indirectly linked to unreliable factors (e.g., national economic growth rates or national population increases). For example, a change in the total population would have an impact on health distress; a change in the population structure would affect social distress; and a change in real estate values or planned land uses would have an impact on economic losses. No parameter ranges are specified, so both extreme and average values can be used by experts and stakeholders. Exposure (directly) and resilience (indirectly) depend on $D E, D U$, and $F R$ according to functions specified by the experts, whereas the levels of $D E, D U$, and $F R$ are chosen by the stakeholders. Second, the records of inputs by experts and stakeholders are saved in order to elicit the relationships between the scores for mitigation or recovery options and values (e.g., a high impact on unemployment) or weights (e.g., the attitude towards risk), where the weights and parameters are set at the mean of the recorded inputs by default. Third, unalike information in Figs. 1 and 2 could be mapped within a (GIS-based) deliberation-support system (i.e., computer-based tools developed to support decision analysis and participatory processes, but also used as a vehicle of communication, training and experimentation, which facilitate dialogue and exchange of information, provide comprehensive insights to non-

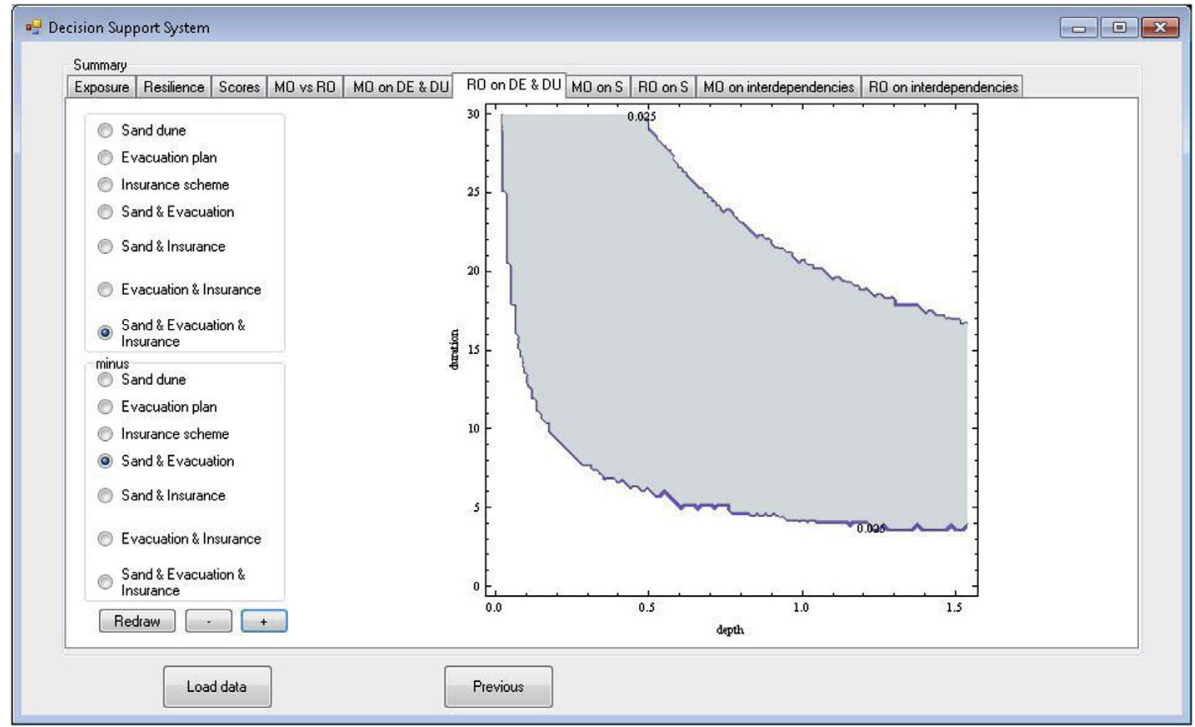

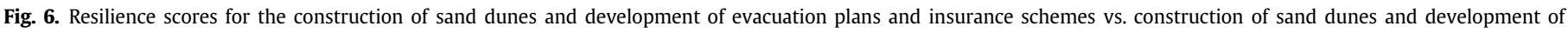
evacuation plans, both as a function of flood depth (m) and duration (days). 


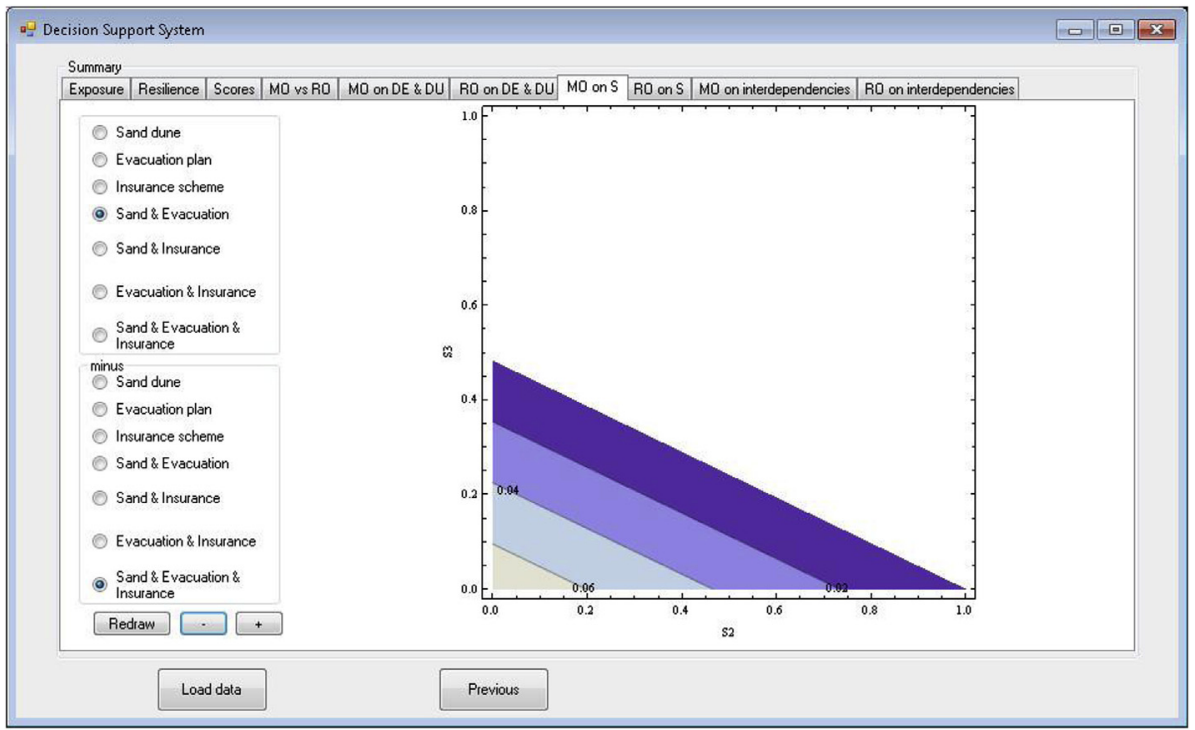

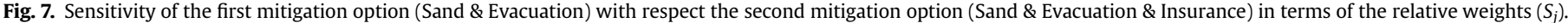
$S_{2}$ and $S_{3}$ represent the relative weights attached to ecological and social features, respectively.

experts, and support them in the exploration of policy options) to show which exposure or resilience indices are most relevant and where they are relevant, as well as which mitigation and recovery options are most efficient and where they are efficient.

In other words, a single combination of mitigation and recovery options could be identified by attaching relative weights to the two objectives of reducing exposure and increasing resilience. This is possible because all qualitative assessments are quantified by applying fuzzy sets, heterogeneous measures are consistently normalised, and interconnected assessments of exposure and resilience are applied.

Note that the most important (free or commercialised) software packages (i.e., Community Vulnerability Assessment Tool CVAT by the National Oceanic and Atmospheric Administration, HAZUS-MH by Federal Emergency Management Agency, Dynamic Interactive Vulnerability Assessment DIVA by the Dinas-Coast consortium, Regional Climate Change Impact and Response
Studies REGIS by East Anglia and North West England; DSSFLOODsite by the FLOODsite Project) are spatial (i.e., they provide maps), but they do not implement interactions between mitigation options nor combinations of qualitative and quantitative impacts. To summarise advantages of our tool with respect to these software packages, let us highlight some features which are peculiar to one software package and which are close to our tool. CVAT provides an overall confidence map, but this is not related to uncertainty on single parameters; HAZUS-MH presents detailed residential and commercial insurance coverage together with flooding costs, but this is not compared with other recovery options; DIVA suggests dikes and nourishment as mitigation options, but these are compared by a Cost Benefit Analysis in monetary terms only; REGIS applies several scenarios, but these are precooked; DSS-FLOODsite distinguishes between resistance and resilience objectives, but related mitigation and recovery options are fixed.

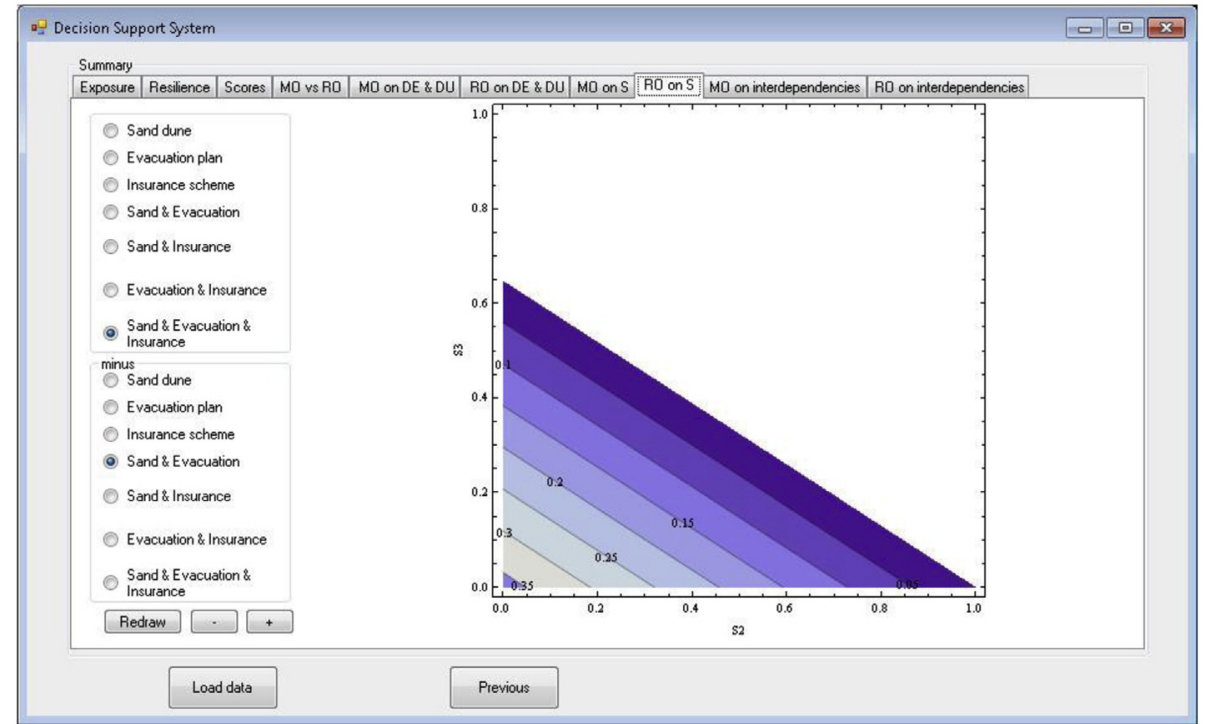

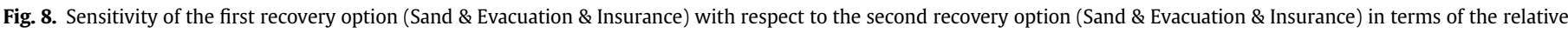
weights $\left(S_{j}\right) . S_{2}$ and $S_{3}$ represent the relative weights attached to ecological and social features, respectively. 


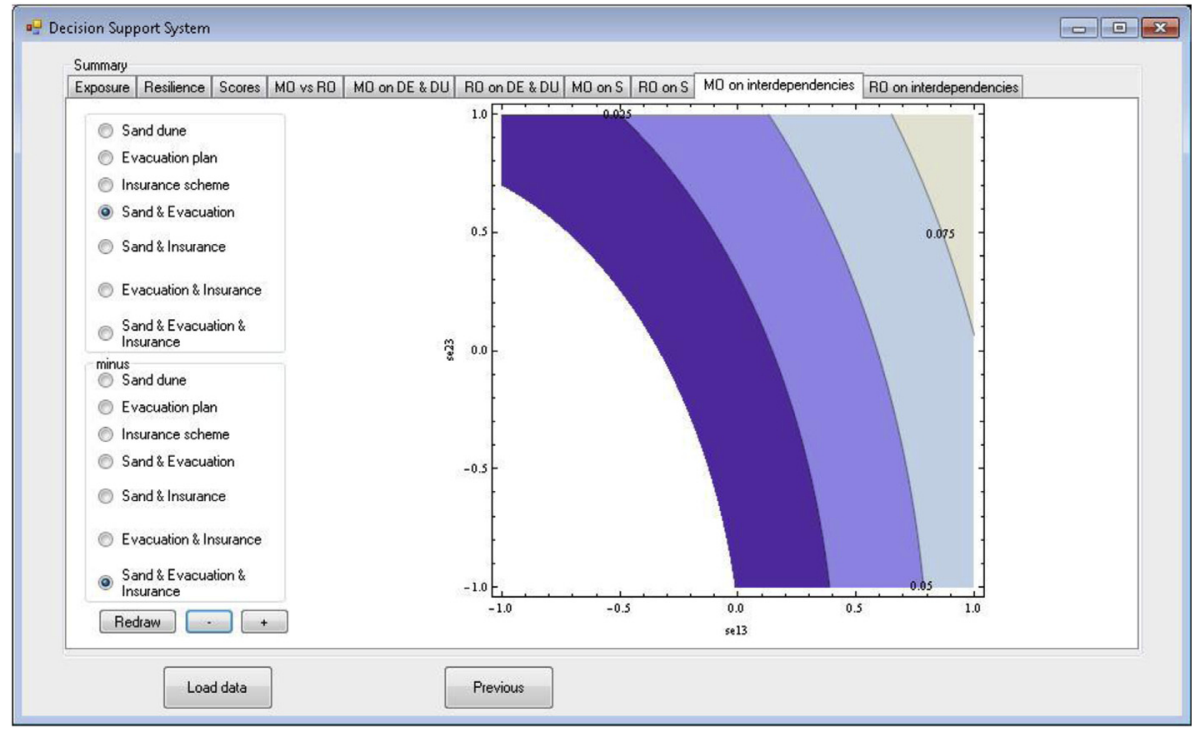

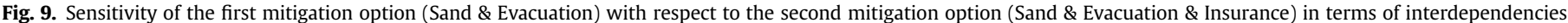
between sand dune construction and development of evacuation plans for health (se13) and social (se23) distresses.

\section{Insights about the case study}

The purpose of this section is twofold. First, we illustrate how a decision is reached using flood depth, duration, and frequency as fixed values or bounded variables by applying some plausible parameters for the case study under consideration. Second, we perform a sensitivity analysis for the decision with respect to the values of some weights and preferences (for given indices and functions) and with respect to the values of some indices and functions (for given weights and preferences).

\subsection{Sample results}

If the flood depth is set at $1 \mathrm{~m}$, the flood duration is set at 20 days (i.e., $2 / 3$ of the maximum value in the model), and the flood frequency is set at 10 (i.e., one flood event every 10 years), and if stakeholders are assumed to be very risk-averse $(Y=0)$, then $I P, L A$, and $I R$ are fixed such that insurance schemes amount to loan subsidies $(I P=L A$ and $I R=0)$, and the best mitigation option becomes construction of sand dunes and development of evacuation plans, whereas the best recovery option turns out to be construction of sand dunes and development of evacuation plans and insurance schemes (Fig. 3).

Fig. 4 shows that for a relative importance attached to exposure with respect to resilience $(Z)$ greater than $95 \%$, the construction of sand dunes and development of evacuation plans has a greater value than the construction of sand dunes and development of evacuation plans and insurance schemes.

Note that since the maximum score is 1 in the theoretical case of extreme relative weights and perfect efficiency of mitigation and recovery options, the values of 0.32 for mitigation options and 0.42 for recovery options mean that combining the construction of sand

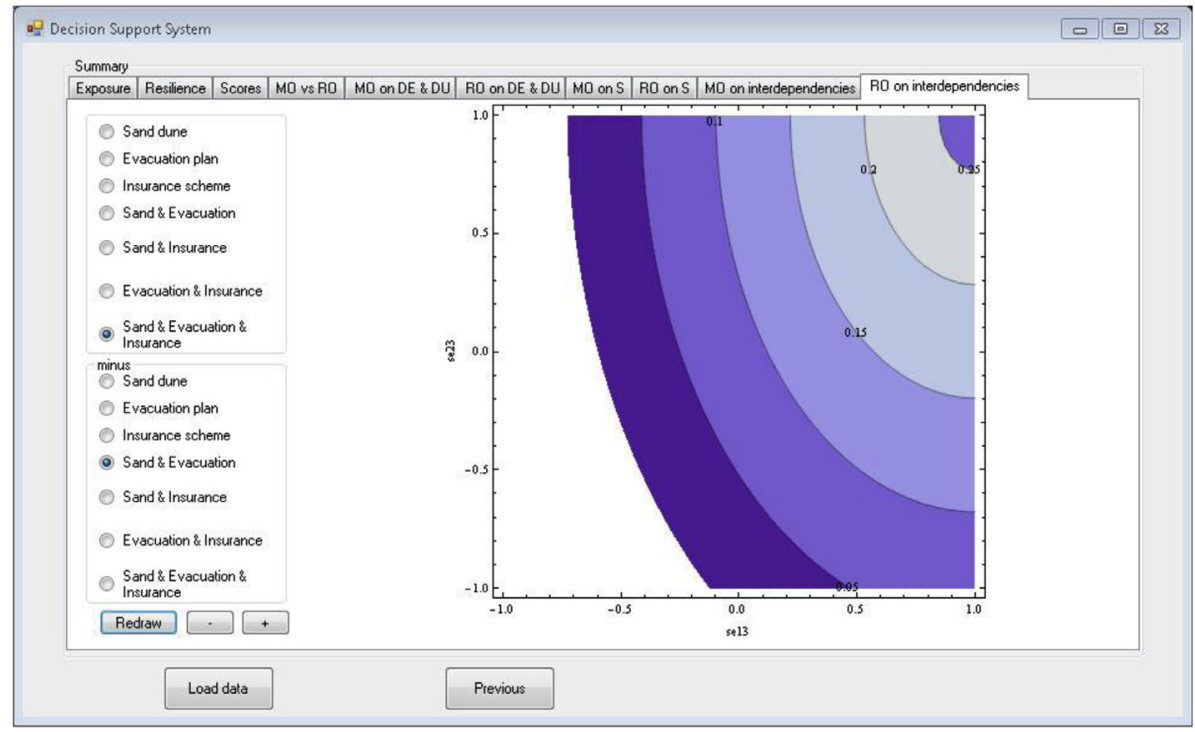

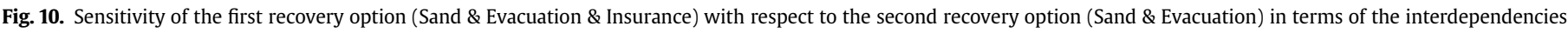
between construction of sand dunes and development of evacuation plans for health (se13) and social (se23) distresses. 
dunes with the development of evacuation plans as mitigation options and the construction of sand dunes and development of evacuation plans and insurance schemes as recovery options would achieve 32 and $42 \%$ of the theoretical maximum score satisfaction, respectively.

In addition to illustrating the overall performance scores of the alternatives considered by the stakeholders and experts, it is important to analyse the robustness of a decision. If the flood depth is within $[0 \mathrm{~m}, 1.5 \mathrm{~m}]$ and the flood duration is within [0 days, 30 days], it turns out that the construction of sand dunes and the development of evacuation plans produces a higher total score than the construction of sand dunes and the development of evacuation plans and of insurance schemes as mitigation options, to a smaller extent for higher levels of flood depth and duration (i.e., the overall performance score decreases), unless flood depth and duration show the highest values (i.e., the overall performance score becomes negative) (Fig. 5); the opposite occurs for recovery options provided that flood depth and duration do not show the lowest or the highest values (Fig. 6).

Note that the specified levels of differences between scores achieved by alternative combinations of mitigation and recovery options can be compared across figures, since they are normalised within $[-1,+1]$.

\subsection{Sensitivity analyses}

The previous section suggests the importance of exploring the sensitivity of the chosen mitigation options and recovery options to simultaneous variations of weights or preferences and indices or functions. Understanding this sensitivity will facilitate the preference-determination process and link the uncertainties in the results to the uncertainties in the different function parameters. The contributions of attitudes towards risk and of individual criteria to the overall performance of the model are not highlighted; no validation is possible, since the DSS refers to future plans, and it would be impractical to perform experiments to test the model's outputs. Refsgaard et al. (2007) noted that many alternative methodologies can be used to recognise uncertainty, including data-uncertainty engines, error-propagation equations, expert elicitation, extended peer review, inverse modelling for parameter estimation or predictive uncertainty, Monte Carlo analysis, multiple-model simulation, quality assurance, scenario analysis, stakeholder involvement, and an uncertainty matrix: we did not examine these options.

Stakeholders are often unsure about how to exactly quantify their preferences. To demonstrate the sensitivity of the model to this uncertainty, we analysed the stability of the results with respect to changes of all weights attached to the criteria by exploring potential trade-offs between conflicting criteria. To do so, we visualised the set of relative weights supporting the chosen mitigation and recovery options by providing a two-dimensional contour plot with given values for flood duration, depth, and frequency, where $S_{2}$ and $S_{3}$ represent the relative weights attached to ecological and social features, respectively. In this plot, positive differences in scores between the chosen options and other alternatives are coloured and mean that the chosen mitigation and recovery options are supported by relative weights specified on the $x$ and $y$-axes, whereas negative differences are white and have an opposite meaning. Note that robustness analysis for potential heterogeneity within each group of experts is not performed explicitly. However, one could check it by changing weights attached to each criterion $\left(E_{i}\right)$, while it could be easily performed explicitly by applying the same procedure used for weights attached to features $\left(S_{j}\right)$.
If relatively small weights are attached to economic features (i.e., relatively large weights are attached to the ecological and social features because the total weights must add up to 1), Fig. 7 shows that the second mitigation option (construction of sand dunes and development of evacuation plans and insurance schemes) could have a greater value than the first mitigation options (construction of sand dunes and development of evacuation plans). Similarly, Fig. 8 shows that construction of sand dunes and development of evacuation plans (the second recovery option) could have a greater value than construction of sand dunes and development of evacuation plans and insurance schemes (the first recovery option). Stakeholders should therefore carefully check whether the weighting accurately reflects their preferences.

Note that this method, which is similar to the triangular plot applied to the multi-attribute value function (e.g., Hodgkin et al., 2005), uses a white area to represent the ranges of relative weights that will produce suboptimal mitigation and recovery options.

Determining the shapes of the value functions is a difficult task in practice. To demonstrate this, we examined the stability of the results with respect to changes in the parameters that represent interdependencies among mitigation and recovery options. To do so, we visualised the set of interdependencies supporting the chosen mitigation and recovery options by providing a twodimensional contour plots with given values for flood duration, depth, and frequency, where $\mathrm{se}_{13}$ and $\mathrm{se}_{23}$ represent the interdependencies between construction of sand dunes and development of evacuation plans in terms of social and health distress, respectively. In this plot, positive differences in scores between the chosen options and other alternatives are coloured and mean that the chosen mitigation and recovery options are supported by relative weights specified on the $x$ - and $y$-axes, whereas negative differences are white and have an opposite meaning.

Fig. 9 shows that construction of sand dunes and development of evacuation plans has a greater value than construction of sand dunes and development of evacuation plans and insurance schemes as a mitigation option if the impact of construction of sand dunes on the overall efficiency of evacuation plans is beneficial in terms of reducing health distress (positive $\mathrm{se}_{13}$ ) or if the impact of construction of sand dunes on health distress is detrimental (negative $\mathrm{se}_{13}$ ) but can be compensated by a large beneficial impact on social distress (positive and large $\mathrm{se}_{23}$ ). In contrast, Fig. 10 shows that the construction of sand dunes and development of evacuation plans and insurance schemes has a greater value than the construction of sand dunes and development of evacuation plans as recovery options if the detrimental impact of construction of sand dunes on the overall efficiency of the evacuation plans in terms of health distress is small (a negative but tiny $\mathrm{se}_{13}$ ) and is compensated by a large beneficial impact of construction of sand dunes on social distress (positive and large $\mathrm{se}_{23}$ ). Thus, the experts should carefully consider the beneficial and detrimental interdependencies among mitigation and recovery options.

Within a multi-expert context, the graphical identification of ranges of parameters supporting the chosen mitigation or recovery options as the optimal solution is more appropriate than presenting relative frequencies of the performance scores of the different alternatives obtained by Monte Carlo simulations (e.g., Bertsch et al., 2007). The advantage of the contour plots is that they intuitively communicate whether a decision can be supported (e.g., it falls within the coloured areas) and to what extent (e.g., the score levels) the decision is supported if the chosen option is good enough (e.g., based on differences in scores). The plots also indicate whether a solution becomes better or worse in response to changes in at least two variables within the set of feasible values (e.g., changes in scores due to changes in parameter values). 


\section{Discussion}

The purpose of this section is to highlight weakness and strengths of the suggested heterogeneous multi-criteria multiexpert multi-objective approach. Note that the DSS presented in this paper is only for the sake of demonstration. In this model, economic, social, and (use) environmental impacts are introduced as economic features (with real numbers and linguistic labels), quantitative and qualitative (non-use) environmental impacts are depicted as ecological features (both with interval numbers), and health and social impacts are introduced as social features (both with linguistic labels). In using the model, one flood measure is suggested by engineers (e.g., construction of sand dunes), one by sociologists (e.g., development of evacuation plans), and one by economists (e.g., insurance schemes or loan subsidies). Moreover, the DSS we presented is designed for prevention (i.e., pro-active planning) rather than for post-crisis responses (i.e., a reactive approach), in terms of both exposure and resilience. The construction of sand dunes has an ex-ante impact on exposure, insurance schemes have an ex-ante impact on resilience, evacuation plans have an ex-post impact on exposure, and loan subsidies have an ex-post impact on resilience. Finally, the DSS is expected to affect risk perception through participatory planning and risk communication. In our analysis, we have not considered the potential costs of these processes, and have instead depicted the dynamics of these processes in terms of the relationships between inputs arising from stakeholder perceptions (e.g., their estimates of flood depth, duration, and frequency) and outputs arising from expert assessments (e.g., calibrated as a function of flood depth, duration, and frequency by the experts).

\subsection{Weaknesses}

Our approach has the following weaknesses:

- It is not a stochastic DSS, but the scenarios depend on continuous values of stochastic variables such as flood depth, duration, and frequency.

- It is not a group DSS that searches for consensus within each group, but crucial groups are nonetheless stakeholders and experts. The approach takes advantage of experts from groups with different knowledge rather than experts with different opinions. The model allows the use of interval values instead of precise values in order to promote the achievement of consensus within groups.

- It is not a fuzzy DSS (based on degrees of confidence or probabilities), and there is no semantic overlapping for linguistic labels or interval numbers, but scenarios can nonetheless be obtained to check for the impacts of alternative parameters.

- It is not a spatial DSS, but allows the choice of alternative combinations of mitigation or recovery options based on the overall net benefits at a municipality level. In our case study, we analysed a single area since using a single source and a single receptor is relevant for our case study area. However, many areas could be studied by attaching relative weights to each area.

- It is not a dynamic DSS, but it does allow consideration of both short-run and long-run time slices.

We used several qualitative indicators, but their values could be determined by quantitative scientific studies. Moreover, awareness of risks could be a target rather than a factor that affects resilience (e.g., in a public information campaign). Finally, the model focuses on efficiency, but it could instead analyse the distribution of flood damage and the costs borne by stakeholders: land uses and values could be linked to stakeholder groups such as property owners, tourism and other entrepreneurs, environmental NGOs, worker or pensioner trade-unions instead of economic, social, and environmental criteria.

\subsection{Strengths}

Our approach has the following strengths:

- Scores are obtained rather than rankings, with the possibility of calculating percentage differences between mitigation and recovery options.

- Sensitivity analyses can be performed graphically for weights and functions, making interpretation of the results more intuitive.

- Stakeholder participation is favoured because users can input the relative weights that should be attached to different features (i.e., economic, social, environmental) or damage functions based on their own risk perception (i.e., flood depth, duration, and frequency). These values could differ from the expert assessments. Records of stakeholder inputs are retained and can be used to evaluate changes in risk perception.

- Expert knowledge is emphasised. The experts define impact functions by considering both short-run and long-run (discounted) impacts, and therefore account for factors that could be misperceived by stakeholders (e.g., species vs. habitats). The choice of flood depth, duration, and frequency by users implicitly refers to long-run or short-run scenarios, but discount rates are not required because the benefits and costs are specified on an annual basis.

- Stakeholders are not expected to express pair-wise comparisons between mitigation and recovery options or criteria.

- Experts are not expected to express pair-wise comparisons (in terms of rankings or interval numbers) between mitigation or recovery options (which are summarised in a decision information matrix with alternatives in rows and criteria in columns), due to the interdisciplinary of flood phenomena; that is, there is no overall score for each mitigation or recovery option for each expert group.

- Some sub-criteria are specific to certain groups to avoid double-counting and to emphasise the value of expertise.

- Apart from the square root form of the damage function, which is the most popular form reported in the literature, all parameters can be changed. Stakeholders could change parameters $a$ and $b$ to depict an (expected) change in risk perception (inclusive of risk awareness and risk concern) in the short-run and in risk preparedness in the long-run. Similarly, experts could change the $v$ parameter to represent an (expected) change in real estate values or planned land uses.

- Interdependencies between mitigation or recovery options are depicted, together with the possible complete ineffectiveness of an option peculiar to a discipline due to the effect of an option from another discipline.

- Both technical innovation (e.g., construction of sand dunes, development of insurance schemes) and social innovation (i.e., new social processes adopted by society so that people change their way of interacting) are depicted. For example, the model allows the reduction of risky behaviour (and so exposure) if insurance schemes link the premium (cost) to self-made devices, and the increase of risk perception (and so resilience) if evacuation plans are implemented by conducting evacuation exercises.

- Both reduced exposure and increased resilience are measured within $[-1,1]$ and depend (directly or indirectly) on $D E, D U$, and $F R$, so that relative weights are introduced to identify 
mitigation or recovery options that can achieve the best combination of these objectives.

Note that economists usually think in terms of exposure, and base their resilience assessment on this parameter. If the exposure, which depends on $D E$ or $D U$ according to the functions specified by the experts, is sufficiently small, then resilience is achieved. In contrast, ecologists usually think in terms of resilience, and base their exposure assessment on this parameter. In this context, resilience is observed if exposure, which depends on $D U$ or $F R$ according to the functions specified by the experts, is sufficiently small. These differences between economists and ecologists are captured in the relative weights specified by the experts, and the consistency of the resilience and exposure measures makes it feasible to compare or combine the two concepts through the relative weights attached to a reduction of exposure and an increase in resilience specified by the stakeholders.

\section{Conclusions}

The innovative operational DSS that we developed is provided here primarily to demonstrate the approach. For example, more options could have been examined or a more detailed and complex set of criteria could have been used. Despite its limitations, three main characteristics of the heterogeneous multi-criteria multiexpert multi-objective approach should be emphasised.

First, by providing an interactive visual tool that facilitates sensitivity analyses and the communication of the modelling results, the DSS does not so much attempt to find an answer as to facilitate the learning process of decision-makers who are considering an issue. The participatory nature of the approach teaches each group about their own perspectives and preferences and those of other stakeholders relating to that issue. Stakeholders are involved in the assessment of relative weights, in the specification of the problem characteristics (flood depth, duration, and frequency), and in the identification of the mitigation or recovery options to be implemented. In other words, the DSS provides an effective way of reflecting on perspectives and of synthesising judgments (Hodgkin et al., 2005).

Second, participation is represented by the weights expressed by stakeholders with respect to economic, environmental, and social impacts, and integration is represented by the relationships between each of these factors and the flood depth, duration, and frequency, which are estimated by experts through functions. In other words, the DSS is a step towards the implementation of a strategic environmental assessment, which has been defined as "a systematic, participatory decision-making support process undertaken to ensure that key factors relating to the environment and sustainability are taken into account in the development of policies, plans, and programmes" (Posas, 2011). The goal of this process is to enhance not only environmental protection but also environmental governance.

Third, a satisficing approach is adopted in which stakeholders (who express preferences and improve their risk perception) and experts (who provide expert advice) work together with decisionmakers (who search for a compromise with a target in mind) to choose a mitigation or recovery option that provides performance at least as good as the alternatives.

Based on these considerations, the heterogeneous form of the information (e.g., both qualitative and quantitative data) arising from the different disciplines that participate in the analysis can be solved with fuzzy sets, the issue of alternative reference points defined by the different disciplines (e.g., improving the status quo for economics and ensuring resilience against change for ecology) can be solved by normalising the values with respect to a single scenario (e.g., the worst or the best status), and the issue of different time horizons can be solved by identifying comparable indices in the short and long runs (e.g., exposure and resilience). In addition, multiple criteria can be combined by using relative weights defined by the stakeholders, and different philosophical approaches can be reconciled by combining different objectives.

Possible future enhancements of the approach include an option for users to switch from a multi-criteria analysis to a cost-benefit analysis, as an alternative method to combine exposure and resilience, based on impacts evaluated by experts in money terms. However, it is not equally satisfactory, due to the unreliability of several monetary values.

\section{Acknowledgements}

We are grateful for the support of the European Commission through the project "Innovative Technologies for safer European coasts in a changing climate" (THESEUS, Contract 244104, FP7.2009-1; www.theseusproject.eu). We thank the project coordinator, Barbara Zanuttigh, of the University of Bologna (Italy), for her patient work.

\section{Appendix A. Supplementary data}

Supplementary data related to this article can be found at http:// dx.doi.org/10.1016/j.envsoft.2013.08.004.

\section{References}

Anisseh, M., Piri, F., Shahraki, M.R., Agamohamadi, F., 2012. Fuzzy extension of TOPSIS model for group decision making under multiple criteria. Artif. Intell. Rev. 38, 325-338.

Bereketli, I., Genevois, M.E., Albayrak, Y.E., Ozyol, M., 2011. WEEE treatment strategies' evaluation using fuzzy LINMAP method. Expert Syst. Appl. 38, 71-79.

Bertsch, V., Treitz, M., Geldermann, J., Rentz, O., 2007. Sensitivity analyses in multiattribute decision support for off-site nuclear emergency and recovery management. Int. J. Energy Sect. Manag. 1, 342-365.

Chamodrakas, I., Martakos, D., 2011. A utility-based fuzzy TOPSIS method for energy efficient network selection in heterogeneous wireless networks. Appl. Soft Comput. 11, 3734-3743.

Chen, T.-Y., Wang, H.-P., Lu, Y.-Y., 2011a. A multi-criteria group decision-making based on interval-valued intuitionistic fuzzy sets: a comparative perspective. Expert Syst. Appl. 38, 7647-7658.

Chen, Y., Li, K.W., Liu, S.-F., 2011b. An OWA-TOPSIS method for multiple criteria decision analysis. Expert Syst. Appl. 38, 5205-5211.

Chu, M.-T., Shyu, J., Tzeng, G.-H., Khosla, R., 2007. Comparison among three analytical methods for knowledge communities group-decision analysis. Expert Syst. Appl. 33, 1011-1024.

Dalalah, D., Hayajneh, M., Batieha, F., 2011. A fuzzy multi-criteria decision making model for supplier selection. Expert Syst. Appl. 38, 8384-8391.

Dheena, P., Mohanraj, G., 2011. Multi-criteria decision-making combining fuzzy set theory, ideal and anti-ideal points for location site selection. Expert Syst. Appl. 38, 13260-13265.

Dowhań, Ł., Wymysłowski, A., Dudek, R., 2009. Multi-objective decision support system in numerical reliability optimisation of modern electronic packaging. Micro-syst. Technol. 15, 1777-1783.

Ekel, P., Queiroz, J., Parreiras, R., Palhares, R., 2009. Fuzzy set based models and methods of multi-criteria group decision-making. Non-linear Anal. 71, e409-e419.

Geldermann, J., Bertsch, V., Treitz, M., French, S., Papamichail, K.N., Hämäläinen, R.P., 2009. Multi-criteria decision support and evaluation of strategies for nuclear remediation management. Omega 37, 238-251.

Guha, D., Chakraborty, D., 2011. Fuzzy multi attribute group decision making method to achieve consensus under consideration of degrees of confidence of experts' opinions. Comput. Ind. Eng. 60, 493-504.

Hahn, E.D., Knott, C.L., 2008. Assessing quality improvement initiatives when expert judgements are uncertain. J. Oper. Res. Soc. 59, 252-258.

Halouani, N., Chabchoub, H., Martel, J.-M., 2009. PROMETHEE-MD-2T method for project selection. Eur. J. Oper. Res. 195, 841-849.

Herrera, F., Martinez, L., Sanchez, P.J., 2005. Managing non-homogeneous information in group decision making. Eur. J. Oper. Res. 166, 115-132.

Hodgkin, J., Belton, V., Koulouri, A., 2005. Supporting the intelligent MCDA user: a case study in multi-person multi-criteria decision support. Eur. J. Oper. Res. 160, 172-189. 
Kain, J.H., Soderberg, H., 2008. Management of complex knowledge in planning for sustainable development: the use of multi-criteria decision aids. Environ. Impact Assess. Rev. 28, 7-21.

Kodikara, P.N., Perera, B.J.C., Kularathna, M.D.U.P., 2010. Stakeholder preference elicitation and modelling in multi-criteria decision analysis - a case study on urban water supply. Eur. J. Oper. Res. 206, 209-220.

Li, D.-F., Huang, Z.-G., Chen, G.-H., 2010. A systematic approach to heterogeneous multi-attribute group decision making. Comput. Ind. Eng. 59, 561-572.

Liu, P., 2011. A weighted aggregation operators multi-attribute group decisionmaking method based on interval-values trapezoidal fuzzy numbers. Expert Syst. Appl. 38, 1053-1060.

McIntosh, B.S., Ascough II, J.C., Twery, M., Chew, J., Elmahdi, A., Haase, D., Harou, J.J. Hepting, D., Cuddy, S., Jakeman, A.J., Chen, S., Kassahun, A., Lautenbach, S., Matthews, K., Merritt, W., Quinn, N.W.T., Rodriguez-Roda, I., Sieber, S., Stavenga, M. Sulis, A., Ticehurstj, J., Volkg, M., Wrobel, M., Van Delden, H., El-Sawahj, S., Rizzoli, A., Voinov, A., 2011. Environmental decision support systems (EDSS) development challenges and best practices. Environ. Modell. Softw. 26, 1389-1402.

Mikhailov, L., Tsvetinov, P., 2004. Evaluation of services using a fuzzy analytic hierarchy process. Appl. Soft Comput. 5, 23-33.

Parreiras, R.O., Ekel, P.Y. Martini, J.S.C., Palhares, R.M., 2010. A flexible consensus scheme for multi-criteria group decision-making under linguistic assessments. Inf. Sci. 180, 1075-1089.

Posas, P.J., 2011. Exploring climate change criteria for strategic environmental assessments. Prog. Plan. 75, 109-154.

Refsgaard, J.C., Van der Sluijs, J.P., Højberg, A.L., Vanrolleghem, P.A., 2007. Uncertainty in the environmental modelling process - a framework and guidance. Environ. Modell. Softw. 22, 1543-1556.
Roghanian, E., Rahimi, J., Ansari, A., 2010. Comparison of first aggregation and last aggregation in fuzzy group TOPSIS. Appl. Math. Model. 34, 3754-3766.

Su, C.-T., Wang, F.-F., 2012. Integrated fuzzy-connective-based aggregation network with real-values genetic algorithm for quality of life evaluation. Neural Comput. Appl. 21, 2127-2135.

Tan, C., 2011. Generalized intuitionistic fuzzy geometric aggregation operator and its application to multi-criteria group decision making. Softw. Comput. 15, $867-876$.

Tsiporkova, E., Boeva, V., 2006. Multi-step ranking of alternatives in a multi-criteria and multi-expert decision making environment. Inf. Sci. 176, 2673-2697.

Vahdani, B., Mousavi, S.M., Tavakkoli-Moghaddam, R., 2011. Group decisionmaking based on novel fuzzy modified TOPSIS method. Appl. Math. Model. 35, 4257-4269.

Van Delden, H., Seppelt, R., White, R., Jakeman, A.J., 2011. A methodology for the design and development of integrated models for policy support. Environ. Modell. Softw. 26, 266-279.

Wu, C.-W., 2009. Decision-making in testing process performance with fuzzy data. Eur. J. Oper. Res. 193, 499-509.

Yan, H.-B., Huynh, V.-N., Nakamori, Y., 2011. A probabilistic model for linguistic multi-expert decision making involving semantic overlapping. Expert Syst. Appl. 38, 8901-8912.

Ye, J., 2011. Fuzzy cross entropy of interval-valued intuitionistic fuzzy sets and its optimal decision-making method based on the weights of alternatives. Expert Syst. Appl. 38, 6179-6183.

Yuen, K.K.F., Lau, H.C.W., 2009. A linguistic possibility-probability aggregation model for decision analysis with imperfect knowledge. Appl. Soft Comput. 9, 575-589. 